\title{
Fragen zur Zertifizierung
}

Nur eine Antwort ist möglich - Einsendeschluss ist der 31. März 2008

1. Wie hoch ist in Deutschland die Anzahl der Gallensteinträger?
a) 100000 .
b) 500000 .
c) 1 Mio.
d) 8-9 Mio.
e) 20 Mio.

2. Mit welchem Keimspektrum ist bei einer akuten Cholezystitis am häufigsten zu rechnen?
a) Salmonellen.
b) Mischinfektionen (Escherichia coli, Streptokokken, Klebsiellen ...).
c) Clostridien.
d) Enterobakterien.
e) Ascaris lumbricoides.

3. Welche Komplikation der akuten Cholezystitis tritt am häufigsten auf?
a) Begleitpankreatitis.
b) Bilio-enterische Fistel.
c) Gallenblasenperforation.
d) Gallenblasenempyem.
e) Gallenblasengangrän.

4. Welches Symptom ist als Leitsymptom der akuten Cholezystitis anzusehen?
a) Rechtsseitiger Oberbauchschmerz.
b) Erbrechen.
c) Fieber.
d) Völlegefühl.
e) Appetitlosigkeit.

5. Welche diagnostische Maßnahme ist die Methode der ersten Wahl zum Ausschluss/Nachweis einer akuten Cholezystitis?
a) Röntgenübersichtaufnahme des Abdomens.
b) Gastroskopie.
c) CT.
$\square$ d) MRCP.
$\square$ e) Sonographie.
$\square$ f) Hepatobiliäre Funktionsszintigraphie.

6. Wie hoch ist in Deutschland der Anteil der Chirurgen, welche bei anamnestischem, laborchemischem und sonographisch hochgradigem Verdacht auf Choledocholithiasis eine ERC mit Steinextraktion vor einer Cholezystektomie vornehmen?
a) $35 \%$.
b) $50 \%$.
c) $70 \%$.
d) $85 \%$.
e) $90 \%$. 


\section{Die akute Cholezystitis}

$\square$ a) ist eine Indikation zur frühelektiven laparoskopischen Cholezystektomie.

b) ist zwingend konservativ zu behandeln.

c) kann nur im beschwerdefreien Intervall von 6 Wochen operativ behandelt werden.

d) ist eine Indikation zur sofortigen operativen Therapie.

e) kann nur durch eine konventionelle Cholezystektomie behandelt werden.

8. In welcher Zeitspanne sollte bei Vorliegen einer akuten Cholezystitis die frühelektive laparoskopische Cholezystektomie durchgeführt werden?

a) Sofort bei Diagnosestellung.

b) $24 \mathrm{~h}$ nach Symptombeginn.

c) $48 \mathrm{~h}$ nach Krankenhauseinweisung.

d) Möglichst $72 \mathrm{~h}$ nach Diagnosestellung.

e) Im Intervall von 6 Wochen.

9. Wie hoch ist der Anteil Schwangerer, welche trotz konservativer Therapie einer akuten Cholezystitis noch in der Schwangerschaft ein Rezidiv erleiden?
a) $5 \%$
b) $10 \%$
c) $20 \%$
d) $30 \%$.
e) $40 \%$.

10. Die ultraschallgestützte perkutane Cholezystostomie ist
a) das Verfahren der Wahl zur Therapie der Gallenblasenperforation.
b) in Einzelfällen für «High-risk»-Patienten geeignet.
c) stets bei Diabetikern mit akuter Cholezystitis einzusetzen.
d) nur bei der akalkulösen Cholezystitis einzusetzen.
e) ein veraltetes Therapieverfahren der akuten Cholezystitis. 